Check for updates

Cite this: J. Mater. Chem. C, 2018, 6, 7257

Received 23rd April 2018, Accepted 19th June 2018

DOI: $10.1039 / c 8 t c 01929 k$

rsc.li/materials-c

\section{Enhanced electrical properties of antimony doped tin oxide thin films deposited via aerosol assisted chemical vapour deposition $\dagger$}

\author{
Sapna D. Ponja, ${ }^{a}$ Benjamin A. D. Williamson, (D) ${ }^{\text {ab }}$ Sanjayan Sathasivam, ${ }^{a}$ \\ David O. Scanlon, (ID ${ }^{\text {abc }}$ Ivan P. Parkin (iD ${ }^{a}$ and Claire J. Carmalt (D) *a
}

\section{Introduction}

The commercial significance of producing efficient and sustainable transparent conducting oxides (TCOs) cannot be underestimated. The excellent optoelectronic properties of TCO thin films are essential for a number of applications including solar cells, gas sensors, catalysis, electronics and optics. ${ }^{1,2}$ Thin films based on tin(Iv) oxide have a wide fundamental band gap $(\sim 3.6 \mathrm{eV})$ and a stability comparable to other TCO systems making them a viable alternative to the more expensive indium-tin oxide (ITO) films. ${ }^{3}$ The enhancement of electrical n-type conductivity properties can be achieved by the introduction of intrinsic defects, including oxygen vacancies, or by introducing extrinsic defects through aliovalent dopants with, for example, higher valency cations. ${ }^{4}$ The electrical resistivity is dependent on the density of charge carriers and their mobility ${ }^{5}$ and for nominally undoped $\mathrm{SnO}_{2}$ resistivity is limited to $10^{-2}-10^{-3} \Omega \mathrm{cm}^{1}$ Dopants such as fluorine and antimony have been used to great effect in improving the n-type conductivity of $\mathrm{SnO}_{2}$ films, ${ }^{6-11}$ with the latter dopant producing blue coloured coatings. However, it is not desirable to dope beyond a certain concentration

\footnotetext{
${ }^{a}$ Materials Chemistry Centre, Department of Chemistry, University College London, 20 Gordon Street, London WC1H OAJ, UK. E-mail: c.j.carmalt@ucl.ac.uk

${ }^{b}$ Thomas Young Centre, University College London, Gower Street, London WC1E 6BT, UK

${ }^{c}$ Diamond Light Source Ltd, Diamond House, Harwell Science and Innovation Campus, Didcot, Oxfordshire OX11 ODE, UK

$\dagger$ Electronic supplementary information (ESI) available. See DOI: 10.1039/ c8tc01929k
}

as this is known to increase electrical resistivity and reduce optical transparency. ${ }^{12,13}$ Three general considerations needed when doping are: (i) the solubility of the dopant in the structure; (ii) shallowness of the dopant level; (iii) the dopant should not behave as a compensatory acceptor (a low energy electron killing defect). ${ }^{14}$ Antimony-doped tin oxide (ATO) materials have received particular attention due to the availability of the constituent metals and a recent increase in the demand for architectural coloured glass. ${ }^{7}$ The production of doped tin oxide films has been achieved by a variety of physical and chemical vapour deposition methods. ${ }^{1,10}$ Aerosol-assisted chemical vapour deposition (AACVD) is a low-cost and versatile technique with the potential to be scaled up for the commercial production of thin films. ${ }^{15}$ Unlike, other methods, AACVD negates the need for highly thermally stable and volatile precursors which widens the choice available for producing films with tuneable properties. ${ }^{16}$

In this paper we doped tin(Iv) oxide with varying concentrations of antimony. The as produced doped $\mathrm{SnO}_{2}$ structure contains $\mathrm{Sb}$ in the +3 and +5 states with $\mathrm{Sb}(\mathrm{v})$ responsible for the bluish colouration of the films. ${ }^{6,8,17}$ Our ATO thin films show significantly enhanced electrical properties with resistivities as low as $4.7 \times 10^{-4}$, and to our knowledge are on par or superior to other published studies, on this material. A computational analysis into the role of intrinsic and Sbrelated defects in $\mathrm{SnO}_{2}$ was carried out using hybrid density functional theory (DFT) and shows at low doping amounts, antimony is indeed incorporated into the lattice in the desired $\mathrm{Sb}(\mathrm{v})$ state. 


\section{Experimental}

\section{Deposition method}

Depositions were carried in an atmosphere of air (BOC). The substrate used was $\mathrm{SiO}_{2}$ barrier coated float glass $(15 \mathrm{~cm} \times 4 \mathrm{~cm} \times$ $0.3 \mathrm{~cm}$; Pilkington Ltd, UK). The coating ( $c a .50 \mathrm{~nm}$ in thickness) prevents the ions within the glass diffusing to the surface. Any surface grease on the glass was removed using a washing regime of detergent, propan-2-ol and acetone. The precursor solution consisting of $0.30 \mathrm{~g}$ butyltin trichloride and dopant amounts of antimony(III) ethoxide (all from Sigma-Aldrich) in $10 \mathrm{~mL}$ methanol was placed in a glass bubbler. An aerosol was generated using a piezo electric device and carried, in a flow of air $\left(1 \mathrm{~L} \mathrm{~min}^{-1}\right)$, through into horizontal bed cold-walled reactor $(15 \mathrm{~cm} \times 5 \mathrm{~cm})$. The glass substrate, placed on the top and bottom of the reactor, was preheated to $450{ }^{\circ} \mathrm{C}$ on a graphite block containing a Whatman cartridge heater monitored by a Pt-Rh thermocouple. Depositions were achieved by the aerosol being carried in a stream of air through a brass baffle into the reactor. Waste vapours left through the exhaust. Once the entire precursor solution had been used up the reactor was allowed to cool to room temperature under air. Complete coverage of the substrate was achieved and the ATO films were blue and transparent with increasing dopant amounts in the precursor solution producing darker films.

\section{Film analysis}

X-ray diffraction (XRD) measurements were obtained using a modified Bruker-Axs D8 diffractometer with parallel beam optics fitted with a PSD LynxEye silicon strip detector to collect diffracted X-ray photons. X-rays were generated using a $\mathrm{Cu}$ source with $\mathrm{Cu} K \alpha 1$ and $\mathrm{Cu} K \alpha 2$ radiation of wavelengths 1.54056 and $1.54439 \AA$, respectively, with an intensity ratio of $2: 1$ and at $40 \mathrm{kV}$ and $30 \mathrm{~mA}$. The incident beam angle was kept at $1^{\circ}$, and the angular range of the patterns collected was $10^{\circ}<$ $2 \theta<66^{\circ}$ with a step size of $0.05^{\circ}$ counted at $4 \mathrm{~s}$ per step. The patterns were analysed for crystallinity and preferred orientation. Peak positions were compared to patterns from the Inorganic Crystal Structure Database (ICDS). The lattice parameters were calculated from powder X-ray diffraction data using the software GSAS and EXPGUI via the Le Bail method.

X-ray photoelectron spectroscopy (XPS) analysis of the films was carried out using a Thermo Scientific K-Alpha spectrometer equipped with a monochromatic $\mathrm{Al}-\mathrm{K} \alpha$ source to identify chemical constituents. The peaks were modelled using CasaXPS software with binding energies adjusted to adventitious carbon $(284.5 \mathrm{eV})$ in order to compensate for the effects of charging. Survey scans were collected in the range $\mathbf{0}-\mathbf{1 3 5 0} \mathrm{eV}$ (binding energy) at a pass energy of $40 \mathrm{eV}$.

Wavelength dispersive X-ray (WDX) analysis was carried out on a Phillips ESEM. The Sn and Sb atomic\% was derived from $\mathrm{Sn}-\mathrm{K} \alpha$ line $(25271.8 \mathrm{eV})$ and $\mathrm{Sb}-\mathrm{K} \alpha(26359.5 \mathrm{eV})$, respectively.

UV/Vis/Near IR transmittance and reflectance spectra were produced using the Perkin Elmer Precisely Lambda 950 spectrometer using an air background and recorded between 320-2500 nm.
Scanning electron microscopy (SEM) was used to determine the film morphology from a top-down configuration using a JEOL JSM-6301F Field Emission instrument with accelerating voltages ranging from 3-5 keV on Au-coated samples. A side-on configuration was achieved using a JEOL JSM-6301F Field Emission instrument with accelerating voltages ranging from $5 \mathrm{keV}$ on Au-coated samples.

Hall effect measurements were carried out on an Ecopia HMS-3000 set up in the Van der Pauw configuration to determine the sheet resistance, free carrier concentration $(N)$ and mobility $(\mu)$. Samples of $1 \mathrm{~cm}^{2}$ were prepared and silver paint (Agar Scientific) was used to form ohmic contacts which were tested on the in-built software prior to measurement. The samples were then subjected to an input current of $1 \mathrm{~mA}$ and a calibrated magnetic field of $0.58 \mathrm{~T}$.

\section{Theoretical methodology}

A computational analysis into the role of intrinsic and Sb-related defects in $\mathrm{SnO}_{2}$ was carried out using ab initio density functional theory (DFT) within the periodic code VASP. ${ }^{18-21}$ All geometrical and electronic optimisations were carried out using the hybrid functional PBE0 formalised by Adamo and Barone. ${ }^{22,23}$ PBE0 adds $25 \%$ of Hartree-Fock exchange to the PBE formalism resulting in an improved treatment of the self-interaction error compared to standard functionals. PBE0 can provide an accurate description of the band gap and geometry of $\mathrm{SnO}_{2}$ compared to experiment, and has been shown to accurately describe the properties of other tin-based oxides. ${ }^{24-33}$ The Projector Augmented Wave method $(\mathrm{PAW})^{34}$ was used to account for the interaction between the core electrons and the valence electrons of each species $(\mathrm{Sn}[\mathrm{Kr}], \mathrm{O}[\mathrm{He}], \mathrm{Sb}[\mathrm{Kr}])$. Initially the conventional unit cell of rutile $\mathrm{SnO}_{2}$ was relaxed using a $400 \mathrm{eV}$ plane-wave cutoff and a $4 \times 4 \times 6 \Gamma$-centred $k$-point mesh to a force convergence tolerance of $0.01 \mathrm{eV}$ atom $^{-1}{ }^{7}$ A supercell expansion of $2 \times 2 \times 3$ (72 atoms) was produced to probe the properties of each defect and its respective charge states. Structural optimisations were undertaken relaxing only the ions within the cell, whilst keeping the cell parameters constant utilising a $\Gamma$-centred $k$-point mesh of $2 \times 2 \times 2$ twinned with a $400 \mathrm{eV}$ plane-wave energy cut-off. All calculations were spin-polarised and convergence was deemed to be complete when the forces acting on all the ions was less than $0.01 \mathrm{eV}$ atom ${ }^{-1} \cdot \mathrm{Sb}_{2} \mathrm{O}_{5}, \mathrm{Sb}_{2} \mathrm{O}_{4}$ and $\mathrm{Sb}_{2} \mathrm{O}_{3}$ were considered limiting phases in the formation of the dopant related species and were optimised using a planewave energy cut-off of $400 \mathrm{eV}$ and $\Gamma$-centred $k$-point meshes of $2 \times 5 \times 4$ (for the 28 -atom $\mathrm{Sb}_{2} \mathrm{O}_{5}$ unit cell), $5 \times 4 \times 2$ (for the 24 atom $\mathrm{Sb}_{2} \mathrm{O}_{4}$ unit cell) and $5 \times 4 \times 2$ (for the 20 atom $\mathrm{Sb}_{2} \mathrm{O}_{3}$ unit cell). The volume, lattice parameters and atoms were allowed to relax until the forces acting on all ions was less than $0.01 \mathrm{eV}^{\text {atom }}{ }^{-1}$.

\section{Defect formalism}

The formation enthalpy for a defect in charge state ' $q$ ' was assessed using the relation:

$$
\begin{aligned}
\Delta H_{\mathrm{f}}(D, q)= & \left(E^{D, q}-E^{\mathrm{H}}\right)+\sum_{i} n\left(E_{i}+\mu_{i}\right) \\
& +q\left(E_{\mathrm{Fermi}}+\varepsilon_{\mathrm{VBM}}^{\mathrm{H}}+\Delta E_{\mathrm{pot}}\right)+q^{2} E_{\mathrm{corr}}^{\mathrm{IC}}+E_{\mathrm{corr}}^{\mathrm{BF}}
\end{aligned}
$$


where $E^{D, q}$ is the total energy of the defective supercell in charge state ' $q$ ' and $E^{\mathrm{H}}$ is the total energy of the host supercell. $E_{i}$ and $\mu_{i}$ correspond to the elemental reference energies and the corresponding chemical potentials respectively (where ' $i$ ' $=\mathrm{Sn}_{(\mathrm{s})}, \mathrm{O}_{2(\mathrm{~g})}$ and $\left.\mathrm{Sb}_{(\mathrm{s})}\right)$. $n$ is either positive or negative depending on whether the element is taken away from or added to the system respectively. $E_{\mathrm{f}}$ is the Fermi energy and ranges from the valence band maximum (VBM) to $\sim 2.4 \mathrm{eV}$ above the conduction band minimum (CBM) which occurs at $3.6 \mathrm{eV} . \varepsilon_{\mathrm{VBM}}^{\mathrm{H}}$ is the eigenvalue of the VBM of the host material and is aligned to the defective supercell via a potential alignment term $\left(\Delta E_{\text {pot }}\right)$ which averages the total potential of the supercell bar that in the immediate vicinity of the defect. ${ }^{35}$

Due to the long ranged nature of the Coulomb interaction $^{36,37}$ an image charge term $q^{2} E_{\text {corr }}^{\mathrm{IC}}$ was applied to account for the interaction of a charged defect and its periodic images. The image charge correction used in this work is based upon the Lany and Zunger formalism ${ }^{35,38}$ with the 'non-cubic' adaptation as implemented by Hine and Murphy. ${ }^{39}$ For shallow and resonant defects, a band-filling correction ( $\left.E_{\text {corr }}^{\mathrm{BF}}\right)$ was applied to regain the dilute limit due to the relatively high carrier concentrations present in supercell calculations. ${ }^{38,40}$

\section{Thermodynamic limits}

A chemical potential range can be applied to account for the extremes in growth conditions; Sn-rich/O-poor and Sn-poor/O-rich. These are set within the bounds of the calculated formation enthalpy of $\mathrm{SnO}_{2}:{ }^{41}$

$$
\mu_{\mathrm{Sn}}+2 \mu_{\mathrm{O}}=\Delta H_{\mathrm{f}}^{\mathrm{SnO}_{2}}=-5.27 \mathrm{eV} \quad(\text { experiment }=-5.98 \mathrm{eV})
$$

A difference between calculation and experiment was expected due to the neglection of entropic effects within the athermal limit which the DFT calculations were performed. Thus the chemical potential limits for $\mathrm{Sn}$ and $\mathrm{O}\left(\mu_{\mathrm{Sn}}\right.$ and $\mu_{\mathrm{O}}$ respectively) for the two growth regimes were therefore:

$$
\begin{aligned}
& \text { Sn-rich/O-poor conditions: } \mu_{\mathrm{Sn}}=0 \mathrm{eV} ; \mu_{\mathrm{O}}=-2.64 \mathrm{eV} \mathrm{s} \\
& \text { Sn-poor/O-rich conditions: } \mu_{\mathrm{Sn}}=-5.27 \mathrm{eV} ; \mu_{\mathrm{O}}=0 \mathrm{eV}
\end{aligned}
$$

Further limitations were imposed on the system due to the formation of dopant related species. Fig. 1 displays the phase stability diagram within the restraints on $\mu_{\mathrm{O}}$ (eqn (2.1) and (2.2)). Under Sn-rich/O-poor conditions $\left(\mu_{\mathrm{O}}=-2.64 \mathrm{eV}\right)$ the limitation on $\mu_{\mathrm{Sb}}$ was based upon the formation of $\mathrm{Sb}_{(\mathrm{s})}$ and therefore $\mu_{\mathrm{Sb}}=0 \mathrm{eV}$. Under Sn-poor/O-rich conditions $\left(\mu_{\mathrm{O}}=0 \mathrm{eV}\right)$, $\mu_{\mathrm{Sb}}$ was limited by the formation of $\mathrm{Sb}_{2} \mathrm{O}_{5}:{ }^{41}$

$$
\mu_{\mathrm{Sb}}+2 \mu_{\mathrm{O}}=\Delta H_{\mathrm{f}}^{\mathrm{Sb}_{2} \mathrm{O}_{5}}=-9.61 \mathrm{eV} \quad(\text { experiment }=-9.97 \mathrm{eV})
$$

Therefore, $\mu_{\mathrm{Sb}}=-4.80 \mathrm{eV}$ under Sn-poor/O-rich conditions.

The thermodynamic transition levels $\left(q / q^{\prime}\right)$ were defined as:

$$
\varepsilon_{\mathrm{D}}\left(q / q^{\prime}\right)=\frac{\Delta H_{\mathrm{f}}(D, q)-\Delta H_{\mathrm{f}}\left(D, q^{\prime}\right)}{q^{\prime}-q}
$$

The evolution of a defect from charge state $q$ to $q^{\prime}$ at a certain Fermi level position can be seen experimentally in techniques such as deep level transient spectroscopy.

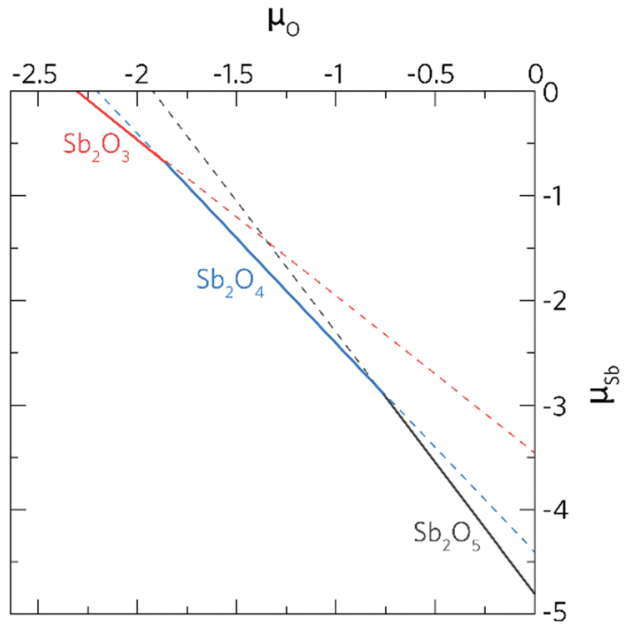

Fig. 1 Phase stability diagram for the Sb-based oxides under varying $\mu_{\circ}$ and $\mu_{\mathrm{Sb}} . \mu_{\mathrm{O}}$ ranges from $0 \mathrm{eV}$ (the formation of $\mathrm{O}_{2(\mathrm{~g})}$ ) to $-2.64 \mathrm{eV}$ (the formation of $\mathrm{Sn}_{(\mathrm{s})}$ ). Under O-rich conditions $\mathrm{Sb}_{2} \mathrm{O}_{5}$ is the limiting phase and under $\mathrm{O}$-poor conditions, $\mathrm{Sb}_{(\mathrm{s})}$ is the limiting phase.

\section{Results and discussion}

The films were deposited via AACVD using butyltin trichloride and antimony(III) ethoxide, when doped, in methanol at $450{ }^{\circ} \mathrm{C}$ using air as the carrier gas. The doped films were blue in coloration, which darkened with increasing dopant concentration. They had excellent coverage across the glass substrate, were adherent (confirmed by the Scotch ${ }^{\mathrm{TM}}$ tape test) and air stable, showing no change over a one year period.

The successful incorporation of $\mathrm{Sb}$ into the $\mathrm{SnO}_{2}$ films was confirmed by wavelength dispersive X-ray spectroscopy (WDX). The results show a positive correlation between the concentration of $\left[\mathrm{Sb}(\mathrm{OEt})_{3}\right]$ in the precursor solution and the concentration in the films giving an average precursor efficiency of $47 \%$.

$\mathrm{X}$-ray diffraction (XRD) carried out on the pure and Sb doped $\mathrm{SnO}_{2}$ films showed them to be phase pure and of the cassiterite phase with no peaks for SbOx observed even at high dopant concentrations therefore suggesting the successful formation of a solid solution (or a significant amorphous component). Le Bail modelling of the XRD patterns showed that doping of $\mathrm{SnO}_{2}$ with $\mathrm{Sb}$ generally results in a contraction of the cassiterite unit cell (Table 1). This follows what is expected for the substitutional doping of $\mathrm{Sb}^{5+}$ as its ionic radii is only $60 \mathrm{pm}$ whereas $\mathrm{Sn}^{4+}$ is $69 \mathrm{pm}$. At high Sb doping concentration (10 at\%) there was an increase in unit cell size compared to 4 and 7 at\%, which was most likely due to incorporation of $\mathrm{Sb}^{3+}$ (radii of $76 \mathrm{pm}$ ) ions as well as the desired $\mathrm{Sb}^{5+}$ into the lattice.

Upon doping a change in the preferred orientation was also seen with doped samples showing less preference for the (101) and (301) planes and more for (002) planes of cassiterite. Doping also caused a slight increase in the crystallite size as estimated using the Deby-Scherrer equation (Table 1). This is not normally observed with the presence of dopants, as the addition of foreign ions into the host lattice usually causes a distortion and hence a decrease in crystallinity. The general trend of increased crystallite size with doping concentration as 
Table 1 The calculated crystallite sizes and Le Bail derived unit cell parameters for the AACVD prepared films

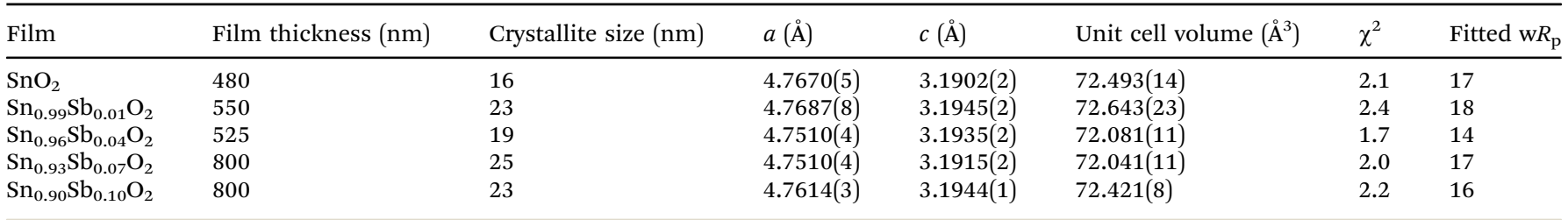

observed here maybe related to the increased film thickness (Fig. 2b-f). As these films were grown on amorphous glass substrates, layers in contact with and close to the substrate would be less crystalline and have smaller crystallite sizes. Due to templating effects the subsequent layers would then grow with larger crystallite sizes as these layers are now growing on crystalline layers beneath them. Therefore thicker films will on average have larger crystallite sizes. This effect has been previously observed for GaAs layers grown on glass via AACVD. ${ }^{42}$ Cross sectional high-resolution transmission electron spectroscopy (HRTEM) on the GaAs films found that crystallites close to the substrate were much smaller in size than the crystallites near the surface of the film.

All the films consist of overlapping tooth like features that are top heavy. It was generally found that thicker films had larger widths of features at the top. Apart from this, the morphology of the films remained fairly consistent with thickness. Notably only one type of crystal growth was seen with no evidence of any amorphous features, strengthening the argument for a complete solid solution.
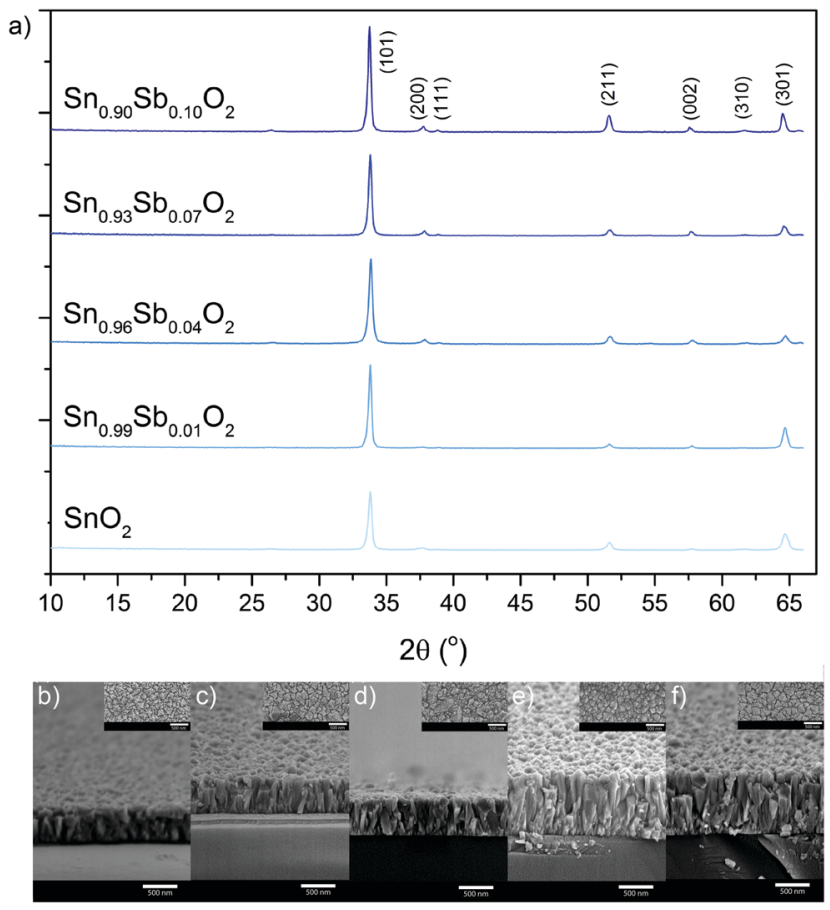

Fig. 2 (a) XRD patterns of the as deposited and nominally Sb doped $\mathrm{SnO}_{2}$ thin films grown via AACVD. (b-f) Show the cross-sectional SEM images with the top down images as inset (scale bar for all images is equal to $500 \mathrm{~nm})$.
X-ray photoelectron spectra (Fig. 3a) showed the $S n 3 d_{5 / 2}$ peak to correspond to literature examples of $\mathrm{Sn}(\mathrm{Iv})$ and to be
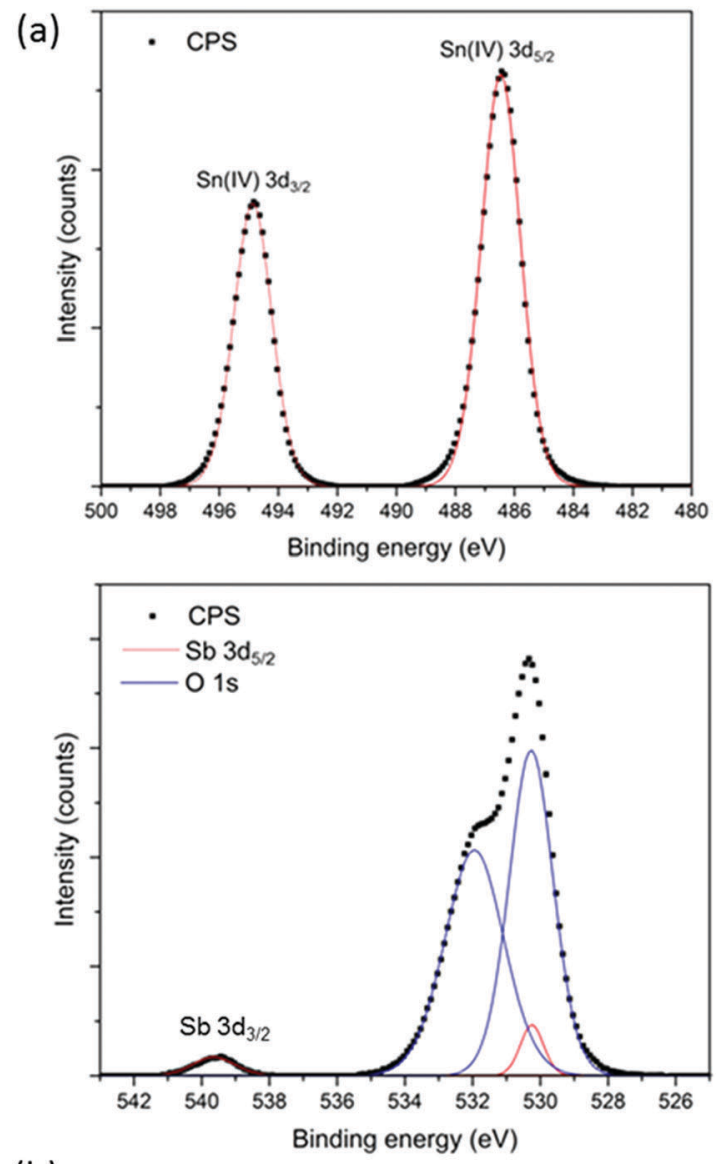

(b)

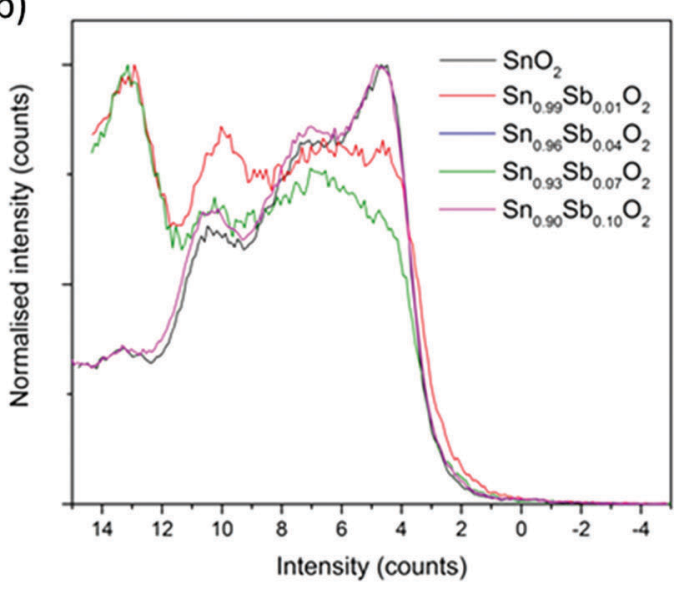

Fig. 3 (a) Core level XPS spectra for the Sn 3d and Sb 3d signals. (b) Valence band spectra for all the AACVD grown films. 
centred at $486.5 \mathrm{eV}$ for all films apart from $\mathrm{Sn}_{0.96} \mathrm{Sb}_{0.04} \mathrm{O}_{2}$ that showed the peak to be slightly shifted to $486.1 \mathrm{eV}$.

The $3 d_{5 / 2}$ peak for $\mathrm{Sb}$ overlaps with the $\mathrm{O} 1 \mathrm{~s}$ but the less intense $3 d_{3 / 2}$ can be used to confirm the presence of $S b$ in the films. The overlapping $\mathrm{Sb} 3 \mathrm{~d}_{5 / 2}$ and $\mathrm{O}$ 1s peak was modelled with a spin orbit split of $9.39 \mathrm{eV}$ and the $\mathrm{Sb} 3 \mathrm{~d}_{5 / 2}$ peak intensity was 1.5 times greater than the $\mathrm{Sb} 3 \mathrm{~d}_{3 / 2}$. Due to the similar peak positions of $\mathrm{Sb}(\mathrm{v})$ and $\mathrm{Sb}(\mathrm{III})$ it is difficult to differenciate between these two oxidation states with certainty from XPS alone, especially at dopant concentrations where signal to noise in the spectra can be low.

Valence band (VB) photoelectron spectra for the pure and $\mathrm{Sb}$ doped $\mathrm{SnO}_{2}$ films are shown in Fig. 3b. All spectra show the three peaks made up of $\mathrm{O} 2 \mathrm{p}$ states that are expected for the VB structure of $\mathrm{SnO}_{2}$. The overall $\mathrm{O} 2 \mathrm{p}$ band width is roughly $9 \mathrm{eV}$. Apart from some peak broadening there was not much change in the VB due to Sb doping. No electronic states were detected within the band gap as a result of doping, this is unusual as normally a peak is expected $0.5 \mathrm{eV}$ below the Fermi level indicating conduction band filling upon semiconduction to metal transition. The absence of this peak maybe due to $\mathrm{Sb}$ being primarily a bulk dopant in the AACVD grown films. This also follows from core level XPS and WDX results that showed $\mathrm{Sb}$ levels at the surface being much lower than in the bulk therefore suggesting bulk segeration.

Hall effect measurements were taken at room temperature using the van der Pauw technique (Table 2). All the films showed n-type conductivity from the negative Hall coefficients. The resistivity, mobility and bulk concentration (charge carrier density) of undoped $\mathrm{SnO}_{2}$ were $1.8 \times 10^{-3} \Omega \mathrm{cm}, 10.4 \mathrm{~cm}^{2} \mathrm{~V}^{-1} \mathrm{~s}^{-1}$ and $3.4 \times 10^{20} \mathrm{~cm}^{-3}$, respectively, values which in general are in the ranges quoted in the literature, albeit, having produced the films through a range of techniques. ${ }^{1,43,44}$ The n-type conductivity is attributed to the formation of shallow donor levels occurring due to the presence of oxygen vacancies and interstitial tin in the $\mathrm{SnO}_{2}$ lattice. ${ }^{44,45}$ While the resistivity increased when 1 at $\% \mathrm{Sb}$ was present in the $\mathrm{SnO}_{2}$ film, a doping level of 4 at\% reduced the resistivity by a factor of 10 . This was associated with an increase in mobility and carrier concentration which can be attributed to the ratio of $\mathrm{Sb}^{3+}$ and $\mathrm{Sb}^{5+}$ present in the lattice. ${ }^{46}$ The $\mathrm{Sb}^{5+}$ state contributes additional free electrons into the conduction band increasing conductivity and the charge carrier concentration. ${ }^{47}$ Above 4 at\% $\mathrm{Sb}$ the resistivity increased with a corresponding decrease in mobility and carrier concentration. In such cases, more of the $\mathrm{Sb}^{5+}$ ions reduce to the $\mathrm{Sb}^{3+}$ state which act as acceptors hence removing electrons from the conduction band.

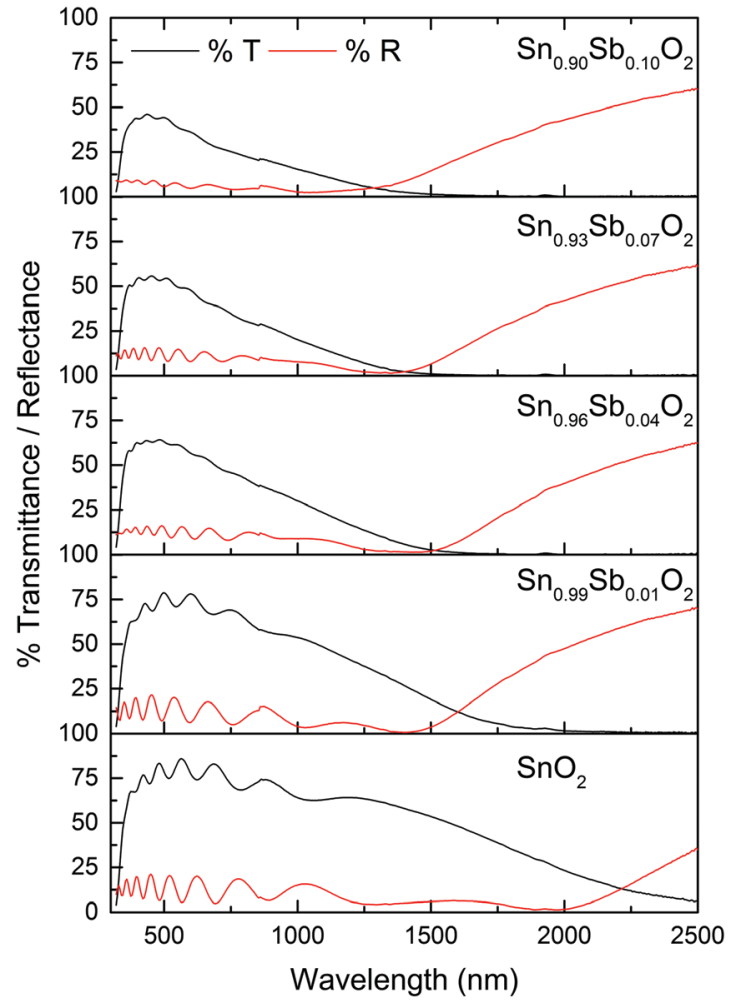

Fig. 4 The transmittance (black) and reflectance (red) spectra of films produced via AACVD.

Scattering of charge carriers limits mobility in polycrystalline materials. ${ }^{47,48}$ A possible reason for the decrease in carrier concentration in excess of 4 at $\%$ is due to the increased disorder that results from the donor having an increased activation energy. ${ }^{47}$

The transmittance and reflectance spectra of the films were collected using visible/near IR spectroscopy between 320 and $2500 \mathrm{~nm}$ and are shown in Fig. 4. The undoped $\mathrm{SnO}_{2}$ film had the highest transmittance of up to $85 \%$ in the visible region. Doping $\mathrm{Sb}$ reduced the optimum transmittance in the visible range and increased reflectance in the infra-red. Transmittance decreased as a function of dopant level (from a maximum of $80 \%$ down to $45 \%)$. Correspondingly, the intensity of the blue colouration of the films increased which is commonly reported. ${ }^{6,17,49}$ However, a variety of explanations have been put forward to account for this observation to fit in with the opto-electronic properties of the films reported. One suggestion is that increasing the Sb dopant amounts leads to an increase

Table 2 The electrical properties of the pure and Sb doped films as determined via room temperature Hall effect measurements

\begin{tabular}{|c|c|c|c|c|c|}
\hline Film & Film thickness (nm) & Sheet resistance $\left(\Omega \mathrm{sq}^{-1}\right)$ & Resistivity, $\rho(\Omega \mathrm{cm})$ & Mobility, $\mu\left(\mathrm{cm}^{2} \mathrm{~V}^{-1} \mathrm{~s}^{-1}\right)$ & $\begin{array}{l}\text { Charge carrier } \\
\text { concentration, } N\left(\mathrm{~cm}^{-3}\right)\end{array}$ \\
\hline $\mathrm{SnO}_{2}$ & 480 & 37.1 & $1.8 \times 10^{-3}$ & 10.4 & $3.4 \times 10^{20}$ \\
\hline $\mathrm{Sn}_{0.99} \mathrm{Sb}_{0.01} \mathrm{O}_{2}$ & 550 & 46.1 & $2.5 \times 10^{-3}$ & 6.9 & $3.6 \times 10^{20}$ \\
\hline $\mathrm{Sn}_{0.96} \mathrm{Sb}_{0.04} \mathrm{O}_{2}$ & 525 & 9.0 & $4.7 \times 10^{-4}$ & 11.4 & $1.2 \times 10^{21}$ \\
\hline $\mathrm{Sn}_{0.93} \mathrm{Sb}_{0.07} \mathrm{O}_{2}$ & 800 & 20.2 & $1.6 \times 10^{-3}$ & 6.2 & $6.2 \times 10^{20}$ \\
\hline $\mathrm{Sn}_{0.90} \mathrm{Sb}_{0.10} \mathrm{O}_{2}$ & 880 & 39.1 & $3.4 \times 10^{-3}$ & 3.1 & $5.8 \times 10^{20}$ \\
\hline
\end{tabular}


in excitable electrons attributed to a greater presence of $\mathrm{Sb}^{5+}{ }^{49}$ In contrast, others suggest, based on the observed reduction in conductivity that the darkening must be due to the presence of an increase in $\mathrm{Sb}^{3+}$ ions concentration. ${ }^{50}$

The plasma edge was estimated from the energy at which the reflectivity reaches half its maximum value. ${ }^{3}$ In general, the plasma band onset of increased reflection shifted towards shorter wavelengths for the doped films compared with pure $\mathrm{SnO}_{2}$.

Emissivity is the ability of a material to emit infrared radiation; values range from 0 for a shiny mirror to 1 for a matt black body. For heat mirror applications reflection in the visible and near infrared are important. A low emissivity value is ideal for a heat mirror as reflection is high in the near infrared. The best commercially available TCO having heat mirror properties is Pilkington's fluorine tin oxide (FTO) having an emissivity value of 0.15 . Emissivity normally drops with increasing dopant concentration reaching a minimum before increasing when more dopant is added. The emissivity of the undoped $\mathrm{SnO}_{2}$ film was 0.33 . Doping with 1 at\% $\mathrm{Sb}$ reduced the emissivity to 0.23 , reaching a minimum of 0.22 at a dopant concentration of 7 at $\%$ and then increasing to 0.30 at the highest dopant concentration.

\section{Computational analysis}

$A b$ initio density functional theory (DFT) was used to determine the role of both intrinsic and $\mathrm{Sb}$ dopant related defects on the electrical properties of $\mathrm{SnO}_{2}$.

The thermodynamic transition levels are displayed in Fig. 5 and show the growth conditions under Sn-rich/O-poor (typically n-type favourable conditions) and Sn-poor/O-rich (p-type preferred conditions) and represent the extreme bounds of the equilibrium growth conditions. The dominant acceptor and donor intrinsic defects were modelled to aid the understanding of the Sb-related defects.

The oxygen and tin vacancies $\left(\mathrm{V}_{\mathrm{O}}\right.$ and $\left.\mathrm{V}_{\mathrm{Sn}}\right)$ represent the dominant intrinsic donor and acceptor defects present in $\mathrm{SnO}_{2}{ }^{24}$ $\mathrm{V}_{\mathrm{O}}$ is a low formation energy deep donor under Sn-rich/O-poor conditions, where the formation energy of the neutral charge state is $\sim 1.89 \mathrm{eV}$ and rises to $\sim 4.53 \mathrm{eV}$ under Sn-poor/O-rich conditions.

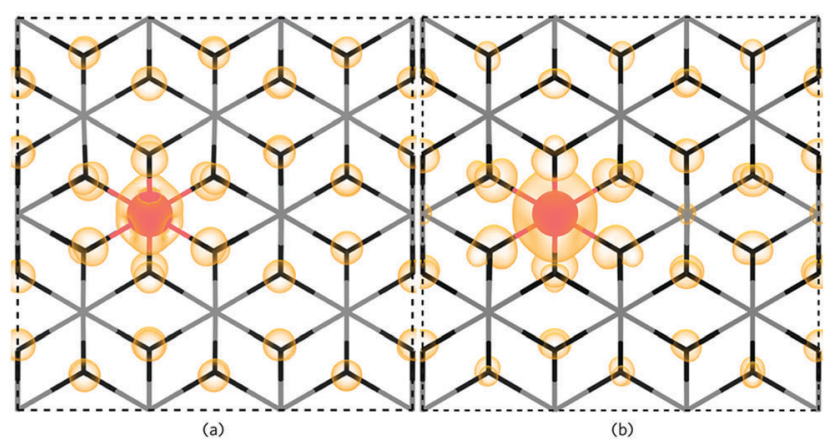

Fig. 5 The thermodynamic transition levels for Sn-rich/O-poor (left) and $\mathrm{Sn}$-poor/O-rich (right). The Fermi energy ranges from the VBM $(0 \mathrm{eV})$ to $\sim 3.4 \mathrm{eV}$ above the CBM. The conduction band is depicted by the graded orange area.
As in other n-type oxides, such as $\mathrm{ZnO}^{31,51-53}$ and $\mathrm{BaSnO}_{3},{ }^{29} \mathrm{~V}_{\mathrm{O}}$ is a negative-U defect ${ }^{32,54-56}$ with the $2+/ 0$ transition level occurring $\sim 0.76 \mathrm{eV}$ below the $\mathrm{CBM}$ in $\mathrm{SnO}_{2}$ revealing that $\mathrm{V}_{\mathrm{O}}$ will not contribute to the conductivity, which is supported by theoretical $^{31,57,58}$ and experimental ${ }^{59,60}$ results alike. $\mathrm{V}_{\text {Sn }}$ also acts as a very deep acceptor with the $0 / 1-$ transition level occurring $\sim 1.75 \mathrm{eV}$ above the VBM. $\mathrm{V}_{\mathrm{Sn}}$ has a very high formation energy over the range of the band gap with the formation energy under the most favourable growth conditions (Sn-poor) being prohibitively high at $\sim 8.37 \mathrm{eV}$.

$\mathrm{Sb}_{\mathrm{Sn}}$ is a resonant donor with the $1+/ 0$ transition level occurring $\sim 0.15 \mathrm{eV}$ above the CBM and has a low formation energy under both Sn-rich/O-poor and Sn-poor/O-rich growth regimes. Interestingly, $\mathrm{Sb}_{\mathrm{Sn}}$ is highest in energy $\left(\Delta H_{\mathrm{f}}\left[\mathrm{Sb}_{\mathrm{Sn}}^{0}\right]=\right.$ $\sim 1.42 \mathrm{eV}$ ) under typically n-type favourable "O-poor" conditions and lowest under a p-type favourable "O-rich" regime $\left(\Delta H_{\mathrm{f}}\left[\mathrm{Sb}_{\mathrm{Sn}}^{0}\right]=\sim 0.95 \mathrm{eV}\right)$. This is due to the lower $\mu_{\mathrm{Sn}}$ under Sn-poor/O-rich conditions and therefore the availability of free $\mathrm{Sn}$ sites. The facile incorporation of $\mathrm{Sb}$, as found with the AACVD films here, is likely due to the similar ionic radii to $\mathrm{Sn}$ $\left(\mathrm{Sn}=0.69 \AA ; \mathrm{Sb}=0.6\left(\mathrm{Sb}^{5+}\right)-0.76\left(\mathrm{Sb}^{3+}\right) \AA\right)^{61}$ and over all charge states $\mathrm{Sb}$ is shown to have little distortion on the rutile lattice. Fig. 6a shows the partial charge density for $\mathrm{Sb}_{\mathrm{Sn}}^{0}$ displaying a localization of the electron density in the $\mathrm{Sb} 5 \mathrm{~s}$ orbital to the $\mathrm{Sb}$ defect. Under both growth conditions, the $0 / 1-$ transition level occurs $\sim 0.41 \mathrm{eV}$ above the CBM and involves an extra electron being trapped on the $\mathrm{Sb} 5 \mathrm{~s}$ orbital forming $\mathrm{Sb}^{3+}$ (Fig. 6b). As the defect states occur at the conduction band minimum there is small localization of density on $\mathrm{O} s$ states and $\mathrm{Sn} \mathrm{s}$ states. Previous hybrid DFT calculations support a resonant low formation energy donor under both conditions, ${ }^{62-64}$ however Lany and co-workers propose that $\mathrm{Sb}$ is a negative $\mathrm{U}$ defect existing in only the $1-$ and $1+$ charge states in contrast to our work. ${ }^{64}$

The $\mathrm{Sb}-\mathrm{O}$ bond lengths increase from $\sim 2.0 \AA$ to $\sim 2.03 \AA$ from the neutral to the $1-$ charge state so little distortion is seen. Under $\mathrm{Sn}$-poor/O-rich conditions, $\mathrm{Sb}_{\mathrm{Sn}}^{0}$ is compensated for by $\mathrm{V}_{\mathrm{Sn}}^{4-} \sim 0.45 \mathrm{eV}$ above the $\mathrm{CBM}$ and at $\sim 2.05 \mathrm{eV}$ under Sn-rich/O-poor conditions. Under both growth extremes therefore, the conductivity and mobilities in ATO films will be limited by the formation of the 0 and 1 - charge state.

Extended X-ray absorption fine structure (EXAFS) have proposed the clustering of $\mathrm{Sb}_{\mathrm{Sn}}$ and $\mathrm{V}_{\mathrm{O}}$ leading to the formation of $\mathrm{Sb}^{3+}$ and a trapped electron in the $\mathrm{V}_{\mathrm{O}}$ site. ${ }^{65,66}\left[\mathrm{Sb}_{\mathrm{Sn}}+\mathrm{V}_{\mathrm{O}}\right]$ clusters were produced for this study in both a 'near' and 'far' configuration to examine this effect. The near configuration involved $\mathrm{Sb}_{\mathrm{Sn}}$ neighbouring a $\mathrm{V}_{\mathrm{O}}$ whilst the far configuration separated the two defects by $\sim 8 \AA$. From our calculations, we find that $\mathrm{Sb}_{\mathrm{Sn}}$ prefers to distort from its $\mathrm{Sn}$ site towards the oxygen vacancy with a binding energy of $\sim 0.78 \mathrm{eV}$. As such, higher Sb doping concentrations will facilitate the association of $\mathrm{Sb}$ with an oxygen vacancy. Typically, Coulombic effects should deter this behaviour from occurring considering that n-type defects should repel each other, however, this clustering is stabilised by the electronic rearrangement to give a lone pair on the $\mathrm{Sb}$ site. $\left[\mathrm{Sb}_{\mathrm{Sn}}+\mathrm{V}_{\mathrm{O}}\right]$ in the far configuration is less energetically favourable than $\left[\mathrm{Sb}_{\mathrm{Sn}}+\mathrm{V}_{\mathrm{O}}\right]$ in the near configuration by $\sim 0.4 \mathrm{eV}$. 

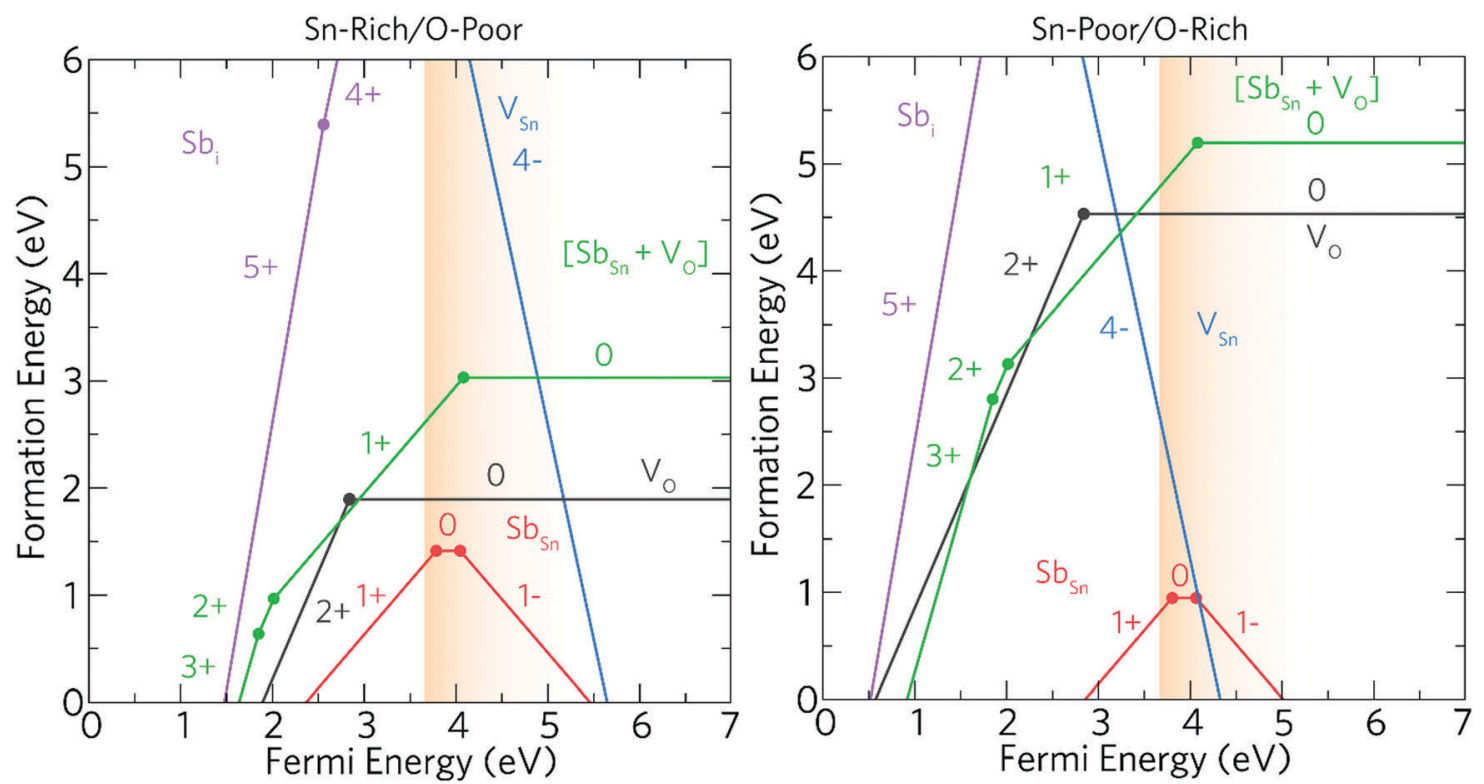

Fig. 6 The partial charge densities for (left) $\mathrm{Sb}_{\mathrm{Sn}}^{0}$ and (right) $\mathrm{Sb}_{\mathrm{Sn}}^{1-}$ as viewed along the (010) direction with a charge density of $0.005 \mathrm{eV} \mathrm{A}^{-1}$. For clarity, the $\mathrm{Sn}$ atoms (grey) and $\mathrm{O}$ atoms (black) are depicted by a stick model and the Sb (red) by a sphere. The electron charge density is shown in orange.

Under Sn-rich/O-poor conditions, $\left[\mathrm{Sb}_{\mathrm{Sn}}+\mathrm{V}_{\mathrm{O}}\right]$ is a shallow donor with a formation energy of $\sim 3.03 \mathrm{eV}$, this rises to $\sim 5.20 \mathrm{eV}$ under an Sn-poor/O-rich regime. $\left[\mathrm{Sb}_{\mathrm{Sn}}+\mathrm{V}_{\mathrm{O}}\right]$ acts as a one electron donor and has two transition levels deep in the bandgap, the $3+/ 2+$ and $2+/ 1+$ transition levels occur $\sim 1.75 \mathrm{eV}$ and $\sim 1.59 \mathrm{eV}$ below the CBM respectively. The $1+/ 0$ transition level occurs $\sim 0.48 \mathrm{eV}$ above the CBM. The partial charge densities for the neutral, $1+$ and $2+$ charge states are shown in Fig. 7a, b and c respectively. For the neutral charge state $\left(\left[\mathrm{Sb}_{\mathrm{Sn}}+\mathrm{V}_{\mathrm{O}}\right]^{0}\right)$, two electrons are located on a lone pair on $\mathrm{Sb}$ (indicating $\mathrm{Sb}(\mathrm{III})$ ) with the remaining electron in the $\mathrm{V}_{\mathrm{O}}$. This is consistent with the EXAFS analysis in which 5-coordinate $\mathrm{Sb}$ is seen corresponding to a removal of a neighbouring oxygen. ${ }^{65,66}$ In the $1+$ charge state an electron is removed from the vacancy site leaving the two electrons in the $\mathrm{Sb}$ lone pair. The $2+$ and $3+$ charge states involve the subsequent removal of electrons from the lone pair as shown in Fig. $7 \mathrm{~b}$ and $\mathrm{c}$. As the $3+$ and $2+$ charge states are very deep in the band gap it is unlikely that these will be seen and as such there will be minimal effect on the optical transparency. It is therefore likely that $\mathrm{Sb}$ (III) will be seen for most situations where the Fermi level is greater than $2.01 \mathrm{eV}$. Sb hardly moves from its position over the range of charge states (maximum distortion of $2 \%$ in all three directions) despite the $\mathrm{Sb}$ ion originally distorting slightly towards the oxygen vacancy.

Under both growth regimes, $\mathrm{Sb}_{\mathrm{i}}$ is a very high formation energy donor, and is unlikely to form at Fermi levels near the CBM. Under Sn-rich/O-poor and Sn-poor/O-rich conditions, the formation energy of $\mathrm{Sb}_{\mathrm{i}}^{0}$ is $\sim 9.40 \mathrm{eV}$ and $\sim 14.20 \mathrm{eV}$ respectively, and so quantities of $S b_{i}$ are expected to be negligible.

$\mathrm{Sb}$ is therefore a low formation energy donor under both doping regimes and is expected to incorporate as $\mathrm{Sb}(\mathrm{v})$ at low doping concentrations. Towards higher concentrations, Sb may either form a localised polaron or likely have a preference to associate with an oxygen vacancy $\left(\mathrm{V}_{\mathrm{O}}\right)$ forming $\mathrm{Sb}(\mathrm{III})$ provided

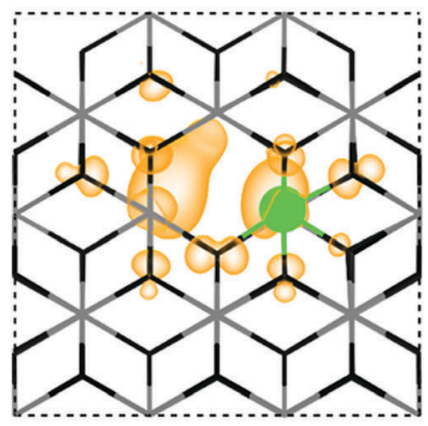

(a)

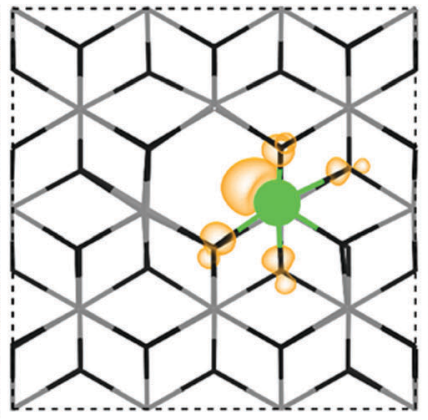

(b)

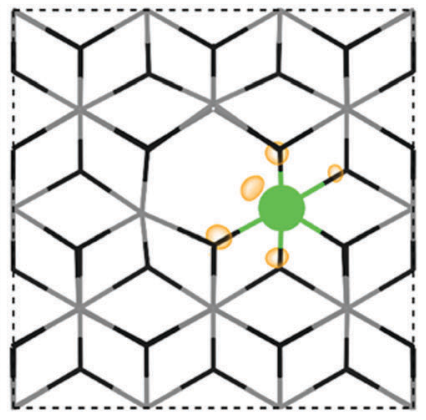

(c)

Fig. 7 The partial charge densities for (a) $\left[S b_{S n}+V_{O}\right]^{0}$ (b) $\left[S b_{S n}+V_{O}\right]^{1+}$ and (c) $\left[S b_{S n}+V_{O}\right]^{2+}$ as viewed along (010) using a charge density of 0.02 eV $A^{-1}$. For clarity, the $\mathrm{Sn}$ atoms (grey) and $\mathrm{O}$ atoms (black) are depicted by a stick model and the Sb (green) by a sphere. The electron charge density is shown in orange. 
the concentration of oxygen vacancies are substantial. This defect complex, however, still donates an electron to the conduction band, accounting for the high conductivities in Sb-doped $\mathrm{SnO}_{2}$ samples here and elsewhere ${ }^{67}$ as well as the prevalence of $\mathrm{Sb}(\mathrm{III})$ and $\mathrm{Sb}(\mathrm{v})$ in the $\mathrm{SnO}_{2}$ lattice.

\section{Conclusion}

The AACVD prepared cassiterite phase pure ATO thin films using simple precursors at $450{ }^{\circ} \mathrm{C}$ show excellent optoelectrical properties. The best performing sample (4 at\% $\mathrm{Sb}$ doped) showed an electrical resistivity of $4.7 \times 10^{-4} \Omega \mathrm{cm}$ resulting from an electron mobility of $11.4 \mathrm{~cm}^{2} \mathrm{~V}^{-1} \mathrm{~s}^{-1}$ and a relatively high carrier density of $1.2 \times 10^{21} \mathrm{~cm}^{-3}$. These results are comparable to some of the lowest resistivity values to date presented in literature for ATO hence having promising technological applications in optoelectronic devices. DFT calculations showed that at low doping concentrations, $\mathrm{Sb}$ incorporates into the $\mathrm{SnO}_{2}$ lattice as $\mathrm{Sb}(\mathrm{v})$. $\mathrm{Sb}(\mathrm{v})$ is a shallow donor with low formation energy. At higher doping regimes, the calculations show that substitutional $\mathrm{Sb}$ reduces to $\mathrm{Sb}$ (III) or associates with oxygen vacancies producing $\mathrm{Sb}$ (III) (present in the AACVD films) acting as a potential limitation to ATO thin films.

\section{Conflicts of interest}

There are no conflicts to declare.

\section{Acknowledgements}

This work made use of the ARCHER UK National Supercomputing Service (http://www.archer.ac.uk) via our membership of the UK's HEC Materials Chemistry Consortium, which is also funded by the EPSRC (EP/L000202). The UCL Legion and Grace HPC Facilities (Legion@UCL and Grace@UCL) were also used in the completion of this work. DOS would like to acknowledge support from the EPSRC (EP/N01572X/1). EPSRC are thanked for a studentship (SDP) and for grant no. EP/L017709. Simon Hurst, of Pilkington NSG, is thanked for emissivity calculations. NSG are thanked for the glass substrates.

\section{References}

1 Y.-Y. Lin, H.-Y. Lee, C.-S. Ku, L.-W. Chou and A. T. Wu, Bandgap narrowing in high dopant tin oxide degenerate thin film produced by atmosphere pressure chemical vapor deposition, Appl. Phys. Lett., 2013, 102(11), 111912.

2 Y. Li, J. Wang, B. Feng, K. Duan and J. Weng, Synthesis and characterization of antimony-doped tin oxide (ATO) nanoparticles with high conductivity using a facile ammoniadiffusion co-precipitation method, J. Alloys Compd., 2015, 634, 37-42.

3 D. S. Bhachu, D. O. Scanlon, G. Sankar, T. D. Veal, R. G. Egdell, G. Cibin, A. J. Dent, C. E. Knapp, C. J. Carmalt and I. P. Parkin, Origin of High Mobility in Molybdenum-Doped Indium Oxide, Chem. Mater., 2015, 27(8), 2788-2796.
4 A. A. Yadav, S. C. Pawar, D. H. Patil and M. D. Ghogare, Properties of (200) oriented, highly conductive $\mathrm{SnO}_{2}$ thin films by chemical spray pyrolysis from non-aqueous medium: effect of antimony doping, J. Alloys Compd., 2015, 652, 145-152.

5 L. Castañeda, Present status of the development and application of transparent conductors oxide thin solid films, Mater. Sci. Appl, 2011, 2, 1233-1242.

6 S. S. Lekshmy, G. P. Daniel and K. Joy, Microstructure and physical properties of sol gel derived $\mathrm{SnO}_{2}: \mathrm{Sb}$ thin films for optoelectronic applications, Appl. Surf. Sci., 2013, 274, 95-100.

7 C. K. T. Chew, C. Salcianu, P. Bishop, C. J. Carmalt and I. P. Parkin, Functional thin film coatings incorporating gold nanoparticles in a transparent conducting fluorine doped tin oxide matrix, J. Mater. Chem. C, 2015, 3(5), 1118-1125.

8 J. P. Correa Baena and A. G. Agrios, Transparent Conducting Aerogels of Antimony-Doped Tin Oxide, ACS Appl. Mater. Interfaces, 2014, 6(21), 19127-19134.

9 K. Ravichandran and P. Philominathan, Analysis of critical doping level of sprayed antimony doped tin oxide films, J. Mater. Sci.: Mater. Electron., 2010, 22(2), 158-161.

10 A. A. Yadav, Influence of film thickness on structural, optical, and electrical properties of spray deposited antimony doped $\mathrm{SnO}_{2}$ thin films, Thin Solid Films, 2015, 591(Part A), 18-24.

11 C. Luan, Z. Zhu, W. Mi and J. Ma, Effect of Sb doping on structural, electrical and optical properties of epitaxial $\mathrm{SnO}_{2}$ films grown on r-cut sapphire, J. Alloys Compd., 2014, 586, 426-430.

12 Y. Jiang, W. Sun, B. Xu, M. Yan and N. Bahlawane, Unusual enhancement in electrical conductivity of tin oxide thin films with zinc doping, Phys. Chem. Chem. Phys., 2011, 13(13), 5760-5763.

13 Z. Banyamin, P. Kelly, G. West and J. Boardman, Electrical and Optical Properties of Fluorine Doped Tin Oxide Thin Films Prepared by Magnetron Sputtering, Coatings, 2014, $4(4), 732$.

14 J. Robertson and B. Falabretti, Electronic Structure of Transparent Conducting Oxides, in Handbook of Transparent Conductors, ed. Ginley, D. S., Springer US, Boston, MA, 2011, pp. 27-50.

15 M. J. Powell and C. J. Carmalt, Aerosols: A Sustainable Route to Functional Materials, Chem. - Eur. J., 2017, 23(62), 15543-15552.

16 P. Marchand, I. A. Hassan, I. P. Parkin and C. J. Carmalt, Aerosol-assisted delivery of precursors for chemical vapour deposition: expanding the scope of CVD for materials fabrication, Dalton Trans., 2013, 42(26), 9406-9422.

17 E. Elangovan and K. Ramamurthi, A study on low cost-high conducting fluorine and antimony-doped tin oxide thin films, Appl. Surf. Sci., 2005, 249(1-4), 183-196.

18 G. Kresse and J. Hafner, Ab initio molecular dynamics for liquid metals, Phys. Rev. B: Condens. Matter Mater. Phys., 1993, 47(1), 558-561.

19 G. Kresse and J. Hafner, Ab Initio Molecular-Dynamics Simulation of the Liquid-Metal-Amorphous-Semiconductor Transition in Germanium, Phys. Rev. B: Condens. Matter Mater. Phys., 1994, 49, 14251. 
20 G. Kresse and J. Furthmüller, Efficiency of ab-initio total energy calculations for metals and semiconductors using a plane-wave basis set, Comput. Mater. Sci., 1996, 6(1), 15-50.

21 G. Kresse and J. Furthmüller, Efficient iterative schemes for ab initio total-energy calculations using a plane-wave basis set, Phys. Rev. B: Condens. Matter Mater. Phys., 1996, 54(16), 11169-11186.

22 C. Adamo and V. Barone, Toward reliable density functional methods without adjustable parameters: the PBE0 model, J. Chem. Phys., 1999, 110(13), 6158-6170.

23 J. Paier, R. Hirschl, M. Marsman and G. Kresse, The PerdewBurke-Ernzerhof exchange-correlation functional applied to the G2-1 test set using a plane-wave basis set, J. Chem. Phys., 2005, 122(23), 234102.

24 D. O. Scanlon and G. W. Watson, On the possibility of p-type $\mathrm{SnO}_{2}$, J. Mater. Chem., 2012, 22(48), 25236-25245.

25 A. M. Ganose and D. O. Scanlon, Band gap and work function tailoring of $\mathrm{SnO}_{2}$ for improved transparent conducting ability in photovoltaics, J. Mater. Chem. C, 2016, 4(7), 1467-1475.

26 S. K. Vasheghani Farahani, T. D. Veal, J. J. Mudd, D. O. Scanlon, G. W. Watson, O. Bierwagen, M. E. White, J. S. Speck and C. F. McConville, Valence-band density of states and surface electron accumulation in epitaxial $\mathrm{SnO}_{2}$ films, Phys. Rev. B: Condens. Matter Mater. Phys., 2014, 90(15), 155413.

27 Z. Lebens-Higgins, D. O. Scanlon, H. Paik, S. Sallis, Y. Nie, M. Uchida, N. F. Quackenbush, M. J. Wahila, G. E. Sterbinsky, D. A. Arena, J. C. Woicik, D. G. Schlom and L. F. J. Piper, Direct Observation of Electrostatically Driven Band Gap Renormalization in a Degenerate Perovskite Transparent Conducting Oxide, Phys. Rev. Lett., 2016, 116(2), 027602.

28 S. Sallis, D. O. Scanlon, S. C. Chae, N. F. Quackenbush, D. A. Fischer, J. C. Woicik, J. H. Guo, S. W. Cheong and L. F. J. Piper, La-doped $\mathrm{BaSnO}_{3}$-Degenerate perovskite transparent conducting oxide: evidence from synchrotron X-ray spectroscopy, Appl. Phys. Lett., 2013, 103(4), 042105.

29 D. O. Scanlon, Defect engineering of BaSnO\$ \{\}$_{-}\{3\} \$$ for highperformance transparent conducting oxide applications, Phys. Rev. B: Condens. Matter Mater. Phys., 2013, 87(16), 161201.

30 J. Suffner, P. Ágoston, J. Kling and H. Hahn, Chemical vapor synthesis of fluorine-doped $\mathrm{SnO}_{2}$ (FTO) nanoparticles, J. Nanopart. Res., 2010, 12(7), 2579-2588.

31 P. Ágoston, K. Albe, R. M. Nieminen and M. J. Puska, Intrinsic n-Type Behavior in Transparent Conducting Oxides: A Comparative Hybrid-Functional Study of $\operatorname{In}_{2} \mathrm{O}_{3}$, $\mathrm{SnO}_{2}$, and ZnO, Phys. Rev. Lett., 2009, 103(24), 245501.

32 J. P. Allen, D. O. Scanlon, L. F. J. Piper and G. W. Watson, Understanding the defect chemistry of tin monoxide, J. Mater. Chem. C, 2013, 1(48), 8194-8208.

33 N. F. Quackenbush, J. P. Allen, D. O. Scanlon, S. Sallis, J. A. Hewlett, A. S. Nandur, B. Chen, K. E. Smith, C. Weiland, D. A. Fischer, J. C. Woicik, B. E. White, G. W. Watson and L. F. J. Piper, Origin of the Bipolar Doping Behavior of SnO from X-ray Spectroscopy and Density Functional Theory, Chem. Mater., 2013, 25(15), 3114-3123.

34 P. E. Blöchl, Projector Augmented-Wave Method, Phys. Rev. B: Condens. Matter Mater. Phys., 1994, 50, 17953.
35 L. Stephan and Z. Alex, Accurate prediction of defect properties in density functional supercell calculations, Modell. Simul. Mater. Sci. Eng., 2009, 17(8), 084002.

36 N. D. M. Hine, K. Frensch, W. M. C. Foulkes and M. W. Finnis, Supercell size scaling of density functional theory formation energies of charged defects, Phys. Rev. B: Condens. Matter Mater. Phys., 2009, 79(2), 024112.

37 M. N. Risto, Issues in first-principles calculations for defects in semiconductors and oxides, Modell. Simul. Mater. Sci. Eng., 2009, 17(8), 084001.

38 S. Lany and A. Zunger, Assessment of correction methods for the band-gap problem and for finite-size effects in supercell defect calculations: case studies for $\mathrm{ZnO}$ and GaAs, Phys. Rev. B: Condens. Matter Mater. Phys., 2008, 78(23), 235104.

39 S. T. Murphy and N. D. M. Hine, Point Defects and Nonstoichiometry in $\mathrm{Li}_{2} \mathrm{TiO}_{3}$, Chem. Mater., 2014, 26(4), 1629-1638.

40 C. Freysoldt, J. Neugebauer and C. G. Van de Walle, Fully Ab Initio Finite-Size Corrections for Charged-Defect Supercell Calculations, Phys. Rev. Lett., 2009, $102(1), 016402$.

41 D. R. Lide, CRC Handbook of Chemistry and Physics, Internet version, CRC Press, Boca Raton, FL, 2005.

42 S. Sathasivam, R. R. Arnepalli, D. S. Bhachu, Y. Lu, J. Buckeridge, D. O. Scanlon, B. Kumar, K. K. Singh, R. J. Visser, C. S. Blackman and C. J. Carmalt, Single Step Solution Processed GaAs Thin Films from $\mathrm{GaMe}_{3}$ and $\mathrm{tBuAsH} 2$ under Ambient Pressure, J. Phys. Chem. C, 2016, 120(13), 7013-7019.

43 A. Oprea, E. Moretton, N. Bârsan, W. J. Becker, J. Wöllenstein and U. Weimar, Conduction model of $\mathrm{SnO}_{2}$ thin films based on conductance and Hall effect measurements, J. Appl. Phys., 2006, 100(3), 033716.

44 K. H. Kim and S. W. Lee, Effect of antimony addition on electrical and optical properties of tin oxide film, J. Am. Ceram. Soc., 1994, 77, 915-921.

45 I. Saadeddin, H. S. Hilal, B. Pecquenard, J. Marcus, A. Mansouri, C. Labrugere, M. A. Subramanian and G. Campet, Simultaneous doping of $\mathrm{Zn}$ and $\mathrm{Sb}$ in $\mathrm{SnO}_{2}$ ceramics: enhancement of electrical conductivity, Solid State Sci., 2006, 8(1), 7-13.

46 S. Gupta, B. C. Yadav, P. K. Dwivedi and B. Das, Microstructural, optical and electrical investigations of $\mathrm{Sb}-\mathrm{SnO}_{2}$ thin films deposited by spray pyrolysis, Mater. Res. Bull., 2013, 48(9), 3315-3322.

47 M. Esro, S. Georgakopoulos, H. Lu, G. Vourlias, A. Krier, W. I. Milne, W. P. Gillin and G. Adamopoulos, Solution processed $\mathrm{SnO}_{2}: \mathrm{Sb}$ transparent conductive oxide as an alternative to indium tin oxide for applications in organic light emitting diodes, J. Mater. Chem. C, 2016, 4(16), 3563-3570.

48 R. Vázquez-Arreguín, M. Aguilar-Frutis, C. FalconyGuajardo, A. Castañeda-Galván, L. Mariscal-Becerra, S. Gallardo-Hernández, G. Alarcón-Flores and M. GarcíaRocha, Electrical, Optical and Structural Properties of $\mathrm{SnO}_{2}: \mathrm{Sb}: \mathrm{F}$ Thin Films Deposited from $\mathrm{Sn}(\mathrm{acac}) 2$ by Spray Pyrolysis, ECS J. Solid State Sci. Technol., 2016, 5(3), Q101-Q107.

49 D.-W. Jung and D.-W. Park, Synthesis of nano-sized antimony-doped tin oxide (ATO) particles using a DC arc plasma jet, Appl. Surf. Sci., 2009, 255(10), 5409-5413. 
50 M. Kojima, H. Kato and M. Gatto, Blackening of tin oxide thin films heavily doped with antimony, Philos. Mag. B, 1993, 68(2), 215-222.

51 F. Oba, M. Choi, A. Togo and I. Tanaka, Point defects in ZnO: an approach from first principles, Sci. Technol. Adv. Mater., 2011, 12(3), 034302.

52 F. Oba, S. R. Nishitani, S. Isotani, H. Adachi and I. Tanaka, Energetics of native defects in ZnO, J. Appl. Phys., 2001, 90(2), 824-828.

53 A. Janotti and C. G. Van de Walle, Native point defects in ZnO, Phys. Rev. B: Condens. Matter Mater. Phys., 2007, 76(16), 165202.

54 A. Walsh, C. R. A. Catlow, A. A. Sokol and S. M. Woodley, Physical Properties, Intrinsic Defects, and Phase Stability of Indium Sesquioxide, Chem. Mater., 2009, 21(20), 4962-4969.

55 S. Lany and A. Zunger, Polaronic hole localization and multiple hole binding of acceptors in oxide wide-gap semiconductors, Phys. Rev. B: Condens. Matter Mater. Phys., 2009, 80(8), 085202.

56 A. Goyal, P. Gorai, H. Peng, S. Lany and V. Stevanović, A computational framework for automation of point defect calculations, Comput. Mater. Sci., 2017, 130(Suppl C), 1-9.

57 P. Ágoston, C. Körber, A. Klein, M. J. Puska, R. M. Nieminen and K. Albe, Limits for n-type doping in $\mathrm{In}_{2} \mathrm{O}_{3}$ and $\mathrm{SnO}_{2}$ : A theoretical approach by first-principles calculations using hybrid-functional methodology, J. Appl. Phys., 2010, 108(5), 053511.

58 A. K. Singh, A. Janotti, M. Scheffler and C. G. Van de Walle, Sources of Electrical Conductivity in $\mathrm{SnO}_{2}$, Phys. Rev. Lett., 2008, 101(5), 055502.

59 S. Samson and C. G. Fonstad, Defect structure and electronic donor levels in stannic oxide crystals, J. Appl. Phys., 1973, 44(10), 4618-4621.
60 P. D. C. King, R. L. Lichti, Y. G. Celebi, J. M. Gil, R. C. Vilão, H. V. Alberto, J. Piroto Duarte, D. J. Payne, R. G. Egdell, I. McKenzie, C. F. McConville, S. F. J. Cox and T. D. Veal, Shallow donor state of hydrogen in $\mathrm{In}_{2} \mathrm{O}_{3}$ and $\mathrm{SnO}_{2}$ : implications for conductivity in transparent conducting oxides, Phys. Rev. B: Condens. Matter Mater. Phys., 2009, 80(8), 081201.

61 R. D. Shannon, Revised effective ionic radii and systematic studies of interatomic distances in halides and chalcogenides, Acta Crystallogr., Sect. A: Cryst. Phys., Diffr., Theor. Gen. Crystallogr., 1976, 32, 751-767.

62 M. Behtash, P. H. Joo, S. Nazir and K. Yang, Electronic structures and formation energies of pentavalent-ion-doped $\mathrm{SnO}_{2}$ : first-principles hybrid functional calculations, J. Appl. Phys., 2015, 117(17), 175101.

63 J. B. Varley, A. Janotti and C. G. Van de Walle, Group-V impurities in $\mathrm{SnO}_{2}$ from first-principles calculations, Phys. Rev. B: Condens. Matter Mater. Phys., 2010, 81(24), 245216.

64 H. Peng, J. D. Perkins and S. Lany, Multivalency of Group 15 Dopants in $\mathrm{SnO}_{2}$, Chem. Mater., 2014, 26(16), 4876-4881.

65 V. Geraldo, V. Briois, L. V. A. Scalvi and C. V. Santilli, EXAFS investigation on $\mathrm{Sb}$ incorporation effects to electrical transport in $\mathrm{SnO}_{2}$ thin films deposited by sol-gel, J. Eur. Ceram. Soc., 2007, 27(13), 4265-4268.

66 J. Rockenberger, U. zum Felde, M. Tischer, L. Tröger, M. Haase and H. Weller, Near edge X-ray absorption fine structure measurements (XANES) and extended x-ray absorption fine structure measurements (EXAFS) of the valence state and coordination of antimony in doped nanocrystalline $\mathrm{SnO}_{2}$, J. Chem. Phys., 2000, 112(9), 4296-4304.

67 K. Y. Rajpure, M. N. Kusumade, M. N. Neumann-Spallart and C. H. Bhosale, Effect of $\mathrm{Sb}$ doping on properties of conductive spray deposited $\mathrm{SnO}_{2}$ thin films, Mater. Chem. Phys., 2000, 64(3), 184-188. 